\title{
Consistency of the EKF-SLAM Algorithm
}

\author{
Tim Bailey, Juan Nieto, Jose Guivant, Michael Stevens and Eduardo Nebot \\ Australian Centre for Field Robotics \\ University of Sydney, NSW, Australia \\ Email: tbailey@acfr.usyd.edu.au
}

\begin{abstract}
This paper presents an analysis of the extended Kalman filter formulation of simultaneous localisation and mapping (EKF-SLAM). We show that the algorithm produces very optimistic estimates once the "true" uncertainty in vehicle heading exceeds a limit. This failure is subtle and cannot, in general, be detected without ground-truth, although a very inconsistent filter may exhibit observable symptoms, such as disproportionately large jumps in the vehicle pose update. Conventional solutions-adding stabilising noise, using an iterated EKF or unscented filter, etc-do not improve the situation. However, if "small" heading uncertainty is maintained, EKF-SLAM exhibits consistent behaviour over an extended time-period. Although the uncertainty estimate slowly becomes optimistic, inconsistency can be mitigated indefinitely by applying tactics such as batch updates or stabilising noise. The manageable degradation of small heading variance SLAM indicates the efficacy of submap methods for large-scale maps.
\end{abstract}

\section{INTRODUCTION}

The original stochastic solution to the SLAM problem by Smith et al. [15] is now almost twenty years old, and the concept has reached a state of maturity sufficient to permit practical implementations in challenging environments. Important considerations that have been addressed are reliable data association in cluttered environments [1], [14]; reduction of computational complexity through locally partitioned datafusion [16], [6] and conservative data-fusion [6], [12], [11]; and bounding accumulated non-linearity with submaps [1], [3]. However, in spite of its clear success in practical applications, the fundamental consistency of the SLAM algorithm has received little attention.

Non-linear SLAM is predominantly implemented as an extended Kalman filter (EKF), where system noise is presumed Gaussian and non-linear models are linearised to suit the Kalman filter algorithm. ${ }^{1}$ EKF-SLAM represents the state uncertainty by an approximate mean and variance. This is a problem in two regards. First, these moments are approximate due to linearisation and may not accurately match the "true" first and second moments. Second, the true probability distribution is non-Gaussian, so that even the true mean and variance may not be an adequate description. These factors affect how the SLAM probability distribution is projected in time, over a sequence of motions and measurements, and how approximation errors accumulate.

Previous work on EKF-SLAM consistency [10], [4], [8] shows that eventual inconsistency of the algorithm is inevitable

\footnotetext{
${ }^{1} \mathrm{~A}$ number of algorithms appearing in the recent literature, such as scan alignment [7] and FastSLAM [13], are built on alternative foundations (e.g., data correlation, particle filters), but these have their own problems, both computational and statistical, and are not considered further in this paper.
}

for large-scale maps, and the estimated uncertainty will become optimistic when compared to the true errors. While this failure due to non-linearity is well-known, no previous work has investigated whether the degree of inconsistency is always significant and under what conditions EKF-SLAM might produce reasonable results.

In this paper, we examine the cause and symptoms of significant inconsistency. We show that vehicle heading uncertainty is a fundamental problem, which precipitates immediate and substantial inconsistency once the "true" heading variance grows sufficiently large. Since heading variance tends to accumulate as the vehicle moves away from the map origin, the problem is inevitable for large maps. Worse, our results indicate that "large variance" is actually quite small, and symptoms of collapse manifest once the true heading standard deviation is above one or two degrees. If heading variance remains small throughout, EKF-SLAM still becomes inconsistent, but it does not exhibit catastrophic failure.

The next section describes the models used in the EKFSLAM simulation experiments, which are performed in the context of a robot with a range-bearing sensor in a 2dimensional environment. Data association is assumed known. Section III examines the symptoms that manifest once EKFSLAM becomes very inconsistent. Section IV demonstrates with Monte Carlo analysis that large heading uncertainty causes gross inconsistency, but that the estimate degrades very slowly if heading variance remains small. Section V discusses the implications of these results and possible solutions to minimise inconsistency, and the final section sums up with concluding remarks.

\section{MODELS FOR EKF-SLAM EXPERIMENTS}

We specify the SLAM state as the vehicle pose (position and heading) and the locations of stationary landmarks observed in the environment. The state at time $k$ is represented by a joint state-vector $\mathbf{x}_{k}$.

$$
\mathbf{x}_{k}=\left[x_{v_{k}}, y_{v_{k}}, \phi_{v_{k}}, x_{1}, y_{1}, \ldots, x_{N}, y_{N}\right]^{T}=\left[\begin{array}{c}
\mathbf{x}_{v_{k}} \\
\mathbf{m}
\end{array}\right]
$$

Notice that the map parameters $\mathbf{m}=\left[x_{1}, y_{1}, \ldots, x_{N}, y_{N}\right]^{T}$ do not have a time subscript as they are modelled as stationary.

To describe the vehicle motion, we use the kinematic model for the trajectory of the front wheel of a bicycle subject to 
rolling motion constraints (i.e., assuming zero wheel slip).

$\mathbf{x}_{v_{k}}=\mathbf{f}_{v}\left(\mathbf{x}_{v_{k-1}}, \mathbf{u}_{k}\right)=\left[\begin{array}{c}x_{v_{k-1}}+V_{k} \Delta T \cos \left(\phi_{v_{k-1}}+\gamma_{k}\right) \\ y_{v_{k-1}}+V_{k} \Delta T \sin \left(\phi_{v_{k-1}}+\gamma_{k}\right) \\ \phi_{v_{k-1}}+\frac{V_{k} \Delta T}{B} \sin \left(\gamma_{k}\right)\end{array}\right.$

Here the time from $k-1$ to $k$ is denoted $\Delta T$, and durit this period the velocity $V_{k}$ and steering angle $\gamma_{k}$ of the fro wheel are assumed constant. Collectively, the velocity a steering values $\mathbf{u}_{k}=\left[V_{k}, \gamma_{k}\right]^{T}$ are termed the "controls". T] wheelbase between the front and rear axles is $B$. The proce model for the joint SLAM state is simply a concatenation the vehicle motion model and the stationary landmark mods

$$
\mathbf{x}_{k}=\mathbf{f}\left(\mathbf{x}_{k-1}, \mathbf{u}_{k}\right)=\left[\begin{array}{c}
\mathbf{f}_{v}\left(\mathbf{x}_{v_{k-1}}, \mathbf{u}_{k}\right) \\
\mathbf{m}
\end{array}\right]
$$

For a range-bearing measurement from the vehicle to landmark $\mathbf{m}_{i}=\left[x_{i}, y_{i}\right]^{T}$, the observation model is given by

$$
\mathbf{z}_{i_{k}}=\mathbf{h}_{i}\left(\mathbf{x}_{k}\right)=\left[\begin{array}{c}
\sqrt{\left(x_{i}-x_{v_{k}}\right)^{2}+\left(y_{i}-y_{v_{k}}\right)^{2}} \\
\arctan \frac{y_{i}-y_{v_{k}}}{x_{i}-x_{v_{k}}}-\phi_{v_{k}}
\end{array}\right]
$$

Adding new landmarks to the map uses an inverse form of the observation model as described in [1, Section 2.2.4] and [10, Section 2]. For the purposes of this paper, the map augmentation model is simply another form of the observation model.

The vehicle motion model, the observation model, and the measured values of the control parameters $\mathbf{u}_{k}$, are not exact, but are subject to noise, which lead to uncertainty in the state estimate. For this reason, we require a probabilistic filter to recursively estimate a distribution over the state given noisy information.

\section{SyMPTOMS OF EKF-SLAM INCONSISTENCY}

The work of Julier and Ulhmann [10] shows that EKFSLAM inevitably becomes inconsistent and that this problem is structural; it cannot be circumvented by adding stabilising noise to the process and observation models. ${ }^{2}$

This inconsistency manifests in two related symptoms. The first is excessive information gain (i.e., reduction in uncertainty), such that the estimated covariance is less than the true covariance. The second is peculiar update characteristics of the state mean, resulting in jumps in the vehicle path and linearly constrained locii of the landmark estimates.

In the following experiments, the process noise is $\left(\sigma_{V}=\right.$ $\left.0.3 \mathrm{~m} / \mathrm{s}, \sigma_{\gamma}=3^{\circ}\right)$ and the observation noise is $\left(\sigma_{r}=\right.$ $0.1 \mathrm{~m}, \sigma_{\theta}=1^{\circ}$ ), unless otherwise stated. The controls are updated every 0.025 seconds and observations occur every 0.2 seconds. The range-bearing sensor has a forward-facing $180^{\circ}$ field-of-view and maximum range of 30 metres.

\footnotetext{
${ }^{2}$ Compensation for inconsistency might be possible by inflating the landmark covariances after each update, but it is not clear how to quantify adequate inflation, and in any case this would nullify all of the SLAM convergence properties described in [5].
}

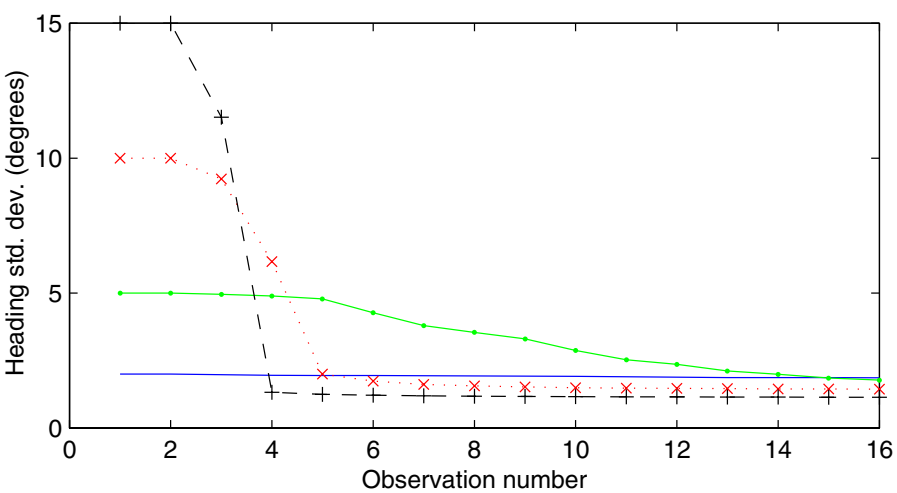

Fig. 1. Information gain in heading for a stationary vehicle. This figure shows the progression of the estimated heading standard deviation $\sigma_{\phi}$ over several observation updates. The results for four different initial values are shown and in each case the estimate should remain constant but exhibits decrease due to linearisation variations.

\section{A. Symptom 1: Information Gain}

When the "true" statistics of the state are available, or can be well approximated, we can compare the true first and second moments $\left(\overline{\mathbf{x}}_{k}, \mathbf{P}_{k}\right)$ with their EKF estimates $\left(\hat{\mathbf{x}}_{k \mid k}, \mathbf{P}_{k \mid k}\right)$. In particular, it is possible to check whether the difference between the true and estimated covariances is positive semidefinite.

$$
\mathbf{P}_{k \mid k}-\mathbf{P}_{k} \geq \mathbf{0}
$$

If it is not then the EKF is optimistic.

To investigate the effect of heading uncertainty on information gain, we first consider a stationary vehicle so that we know throughout the true statistics, and subsequently examine information gain for a moving vehicle. These experiments are essentially the same as in [10], but with a focus on the influence of heading variance.

1) Stationary Vehicle: Consider a stationary vehicle with an initial pose estimate

$$
\begin{aligned}
\hat{\mathbf{x}}_{0 \mid 0} & =[0,0,0]^{T} \\
\mathbf{P}_{0 \mid 0} & =\operatorname{diag}\left(\sigma_{x}^{2}, \sigma_{y}^{2}, \sigma_{\phi}^{2}\right)
\end{aligned}
$$

The vehicle subsequently observes a single landmark and adds it to the SLAM-map. Clearly, the true uncertainty of the landmark can never fall below the initial uncertainty of the vehicle pose. Similarly, the true uncertainty of the vehicle pose may never decrease, regardless of how many times the landmark is observed.

For the EKF-SLAM algorithm, the covariance in vehicle position $\left(\sigma_{x}^{2}, \sigma_{y}^{2}\right)$ is correct; it does not change from the initial value. However, the heading variance $\sigma_{\phi}^{2}$ shows an immediate and dramatic decrease, with the quantity of information gained being dependent on the initial heading variance. The results in Fig. 1 show the spurious information gain for a range of initial heading uncertainties. The gain is dependent on the actual observation errors, and so varies from run to run. In Fig. 1, each line represents the worst-case run of 50 Monte Carlo simulations. Notice that the larger initial uncertainties tend to produce the smallest final uncertainty estimates. 
The gain of information for a stationary vehicle is entirely due to variation in the observation Jacobian $\frac{\partial \mathbf{h}}{\partial \mathbf{x}_{k}}$ over the observation sequence. If the Jacobian were to remain fixed (e.g., linearised about the true state), there would be no loss in uncertainty. ${ }^{3}$ For a normal EKF run, however, the amount of spurious information gained tends to be greater with increased observation uncertainty $\mathbf{R}_{k}$ and, more significantly, with larger initial heading variance $\sigma_{\phi}^{2}$. (Information gain is completely independent of vehicle position variance $\sigma_{x}^{2}$ and $\sigma_{y}^{2}$, which is somewhat counter-intuitive since the observation model is non-linear in $x_{v}, y_{v}$ and linear in $\phi_{v}$.)

This result exemplifies several important points. First, that the EKF-SLAM update step is always inconsistent for any finite values of $\mathbf{R}_{k}$ and $\sigma_{\phi}^{2}$. An algebraic proof of this is provided in [10, Section 3]. Second, the quantity of spurious information gained decreases as "true" heading variance decreases, both in absolute terms and in relation to $\sigma_{\phi}^{2}$. If heading variance is large, the effect of an observation update is immediate and catastropic; if heading variance is small, the inconsistency is tiny and accumulates very slowly. The highly nonlinear relationship between true heading variance and information gain is key to the findings in this paper.

2) Moving Vehicle: To examine information gain for EKFSLAM with a moving vehicle, we compare the results from a nominal EKF run with those obtained using "ideal Jacobians", whereby the process and observation models are linearised about the true (or ideal) values for the state $\mathbf{x}_{k}$ and controls $\mathbf{u}_{k}$. While using ideal Jacobians may not ensure true statistics, our results show that they do provide consistent estimates in terms of the NEES (see Section IV), and the difference between ideal and EKF Jacobian results is a good indicator of nominal EKF performance.

Fig. 2 shows the result of the two runs. Notice that the covariance estimates of the standard EKF are smaller than for the EKF with ideal Jacobians. This indicates a significant level of information gain in the standard algorithm. ${ }^{4}$ The information gain in heading is shown in Fig. 3, where the top line is the ideal Jacobian estimate and the lower line is the standard EKF estimate. This result corroborates with the findings of Castellanos et al. [4]. The heading uncertainty for the ideal Jacobian solution grows while ever the vehicle travels further into unmapped territory but, for the standard EKF solution, the heading uncertainty reaches a ceiling and levels off. Here, the information gain is negligible while the

\footnotetext{
${ }^{3}$ Notice in Fig. 1 that, in each case, there is no spurious information gain until the third observation. The first observation initialises the landmark, the second updates the landmark estimate using the same linearisation values as the first, and the third observation is the first to incorporate a conflicting Jacobian.

${ }^{4}$ It is interesting to observe in Fig. 2 that the standard EKF estimate appears to be more accurate than the ideal Jacobian estimate. This is purely coincidence, and the standard algorithm is, in fact, inconsistent; the crosscorrelation terms of the state covariance are wrong, and may manifest with large estimate errors at some later time. We ran a series of Monte Carlo simulations of the scenario in Fig. 2, using the standard EKF with the same noise characteristics, and the majority of runs produce estimates that are clearly optimistic. The example chosen in Fig. 2(a) is intended to show that a single run with small error in the state mean does not necessarily indicate that the filter is consistent.
}

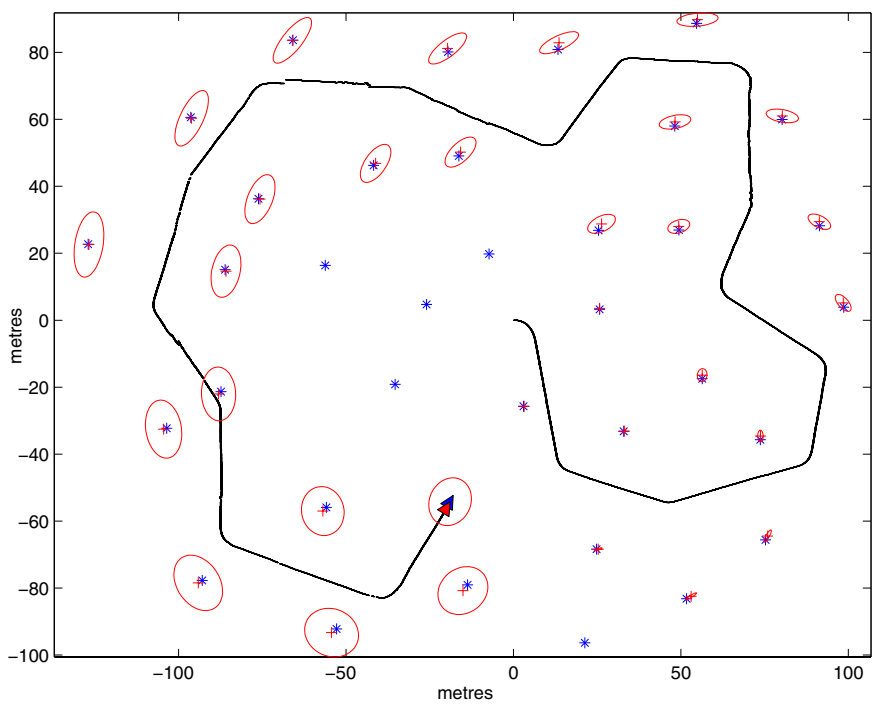

(a) Jacobians linearised about the estimated state.

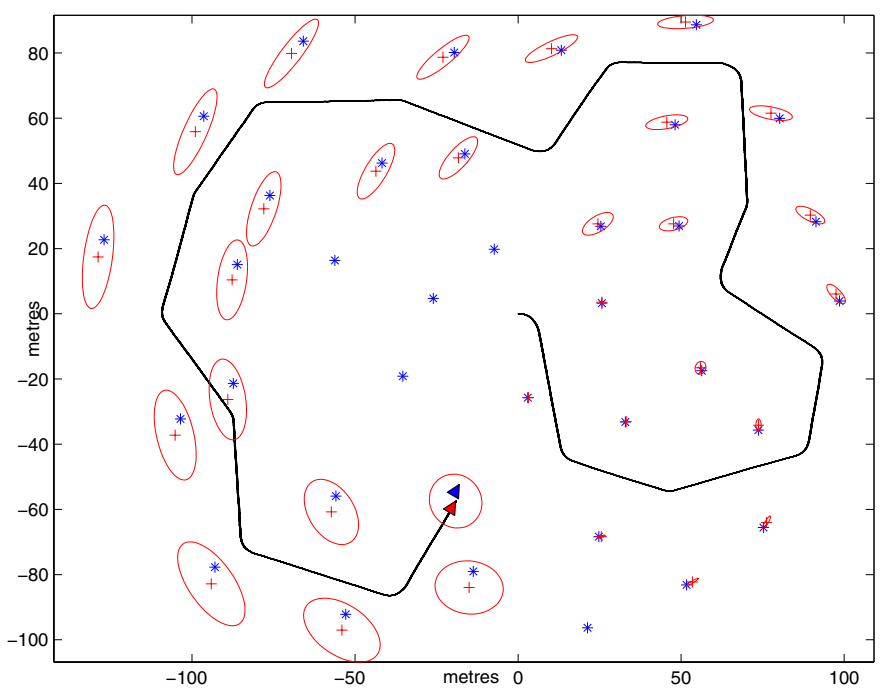

(b) Jacobians linearised about the true state.

Fig. 2. EKF-SLAM simulations showing the estimated vehicle trajectory and 2 -sigma ellipses of landmark locations. The true landmarks are shown as $*$.

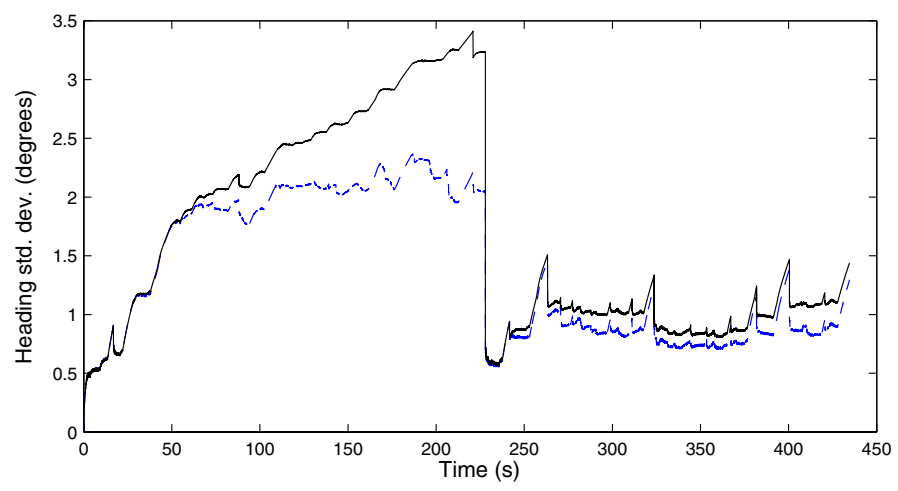

Fig. 3. EKF-SLAM heading uncertainty. The vehicle performs two loops of the trajectory shown in Fig. 2. The large decrease in uncertainty at 220 seconds is when the vehicle first closes the loop. 
heading standard deviation remains below about 1.7 degrees, but rapidly becomes significant at around 2 degrees.

We found that if the standard EKF-SLAM algorithm is run with direct observation of the heading at regular intervals, so that the heading uncertainty is always small, the estimated covariance is virtually identical to the ideal Jacobian result. That is, the information gain for EKF-SLAM with small heading uncertainty is negligible. This property is insensitive to the values of process and observation noise.

\section{B. Symptom 2: Jagged Vehicle Trajectory}

The gross inconsistency of the EKF-SLAM algorithm becomes visibly evident with the appearance of strange discontinuities in the estimated vehicle trajectory. Over successive observation updates, the mean estimate of the vehicle pose shows dramatic jumps, which tend to be disproportionately large when compared to the size of the actual measurement error. A related symptom appears in the locii of landmark location estimates. Rather than exhibiting random motion in accord with the sensor noise, the landmark mean updates seem to be constrained to a line. Again, the size of the update tends to be much larger than the actual measurement error. These symptoms manifest once the vehicle heading uncertainty becomes "large", but do not appear if the Jacobians are linearised about the true state (see Fig. 4). Note that these symptoms are not due to numerical errors; different numerical forms of the EKF give identical results. ${ }^{5}$

It is difficult to say precisely why these symptoms appear. The information gain in heading induces over-strong correlations between the vehicle and the landmarks. Perhaps these invalid cross-correlations constrain the feature updates to follow linear paths, and measurements that attempt to shift a landmark estimate orthogonal to a certain line result in large updates along the line and large jumps in vehicle pose to compensate. Perhaps, on the other hand, these symptoms are simply due to a measurement trying to correct the vehicle heading, when the heading variance has been artificially reduced.

\section{Monte Carlo Tests of Filter Consistency}

When the "true" probability density function is not available, but the true state $\mathbf{x}_{k}$ is known, we can use the normalised estimation error squared (NEES) [2, page 234] to characterise the filter performance.

$$
\epsilon_{k}=\left(\mathbf{x}_{k}-\hat{\mathbf{x}}_{k \mid k}\right)^{T} \mathbf{P}_{k \mid k}^{-1}\left(\mathbf{x}_{k}-\hat{\mathbf{x}}_{k \mid k}\right)
$$

\footnotetext{
${ }^{5}$ We tested several different numerical implementations of the EKF (i.e., a simple covariance form, a Cholesky decomposed form, and numerically and analytically derived Jacobians) and obtained identical results for identical runs (i.e., runs where the random number generator is initialised with the same seed). We would expect some variation if numerical errors were to blame.
}

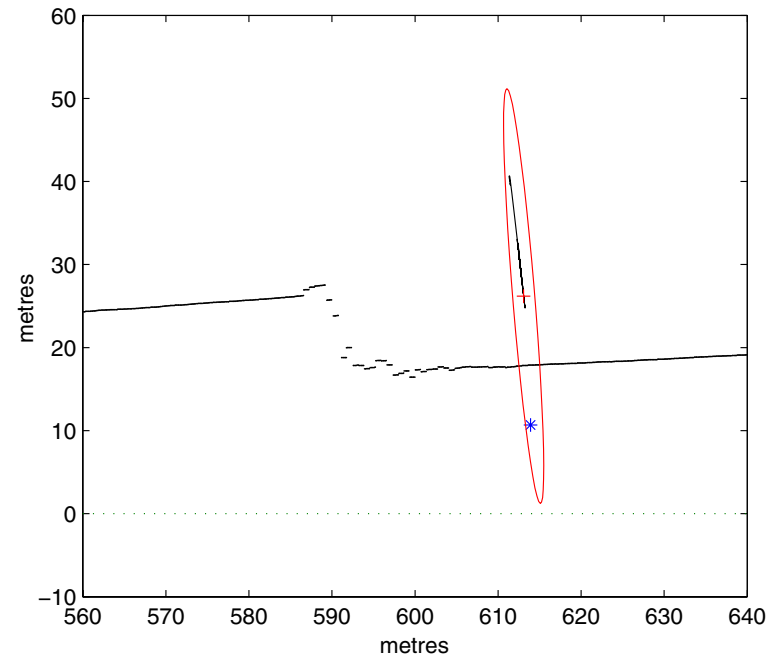

(a) Normal EKF Jacobians.

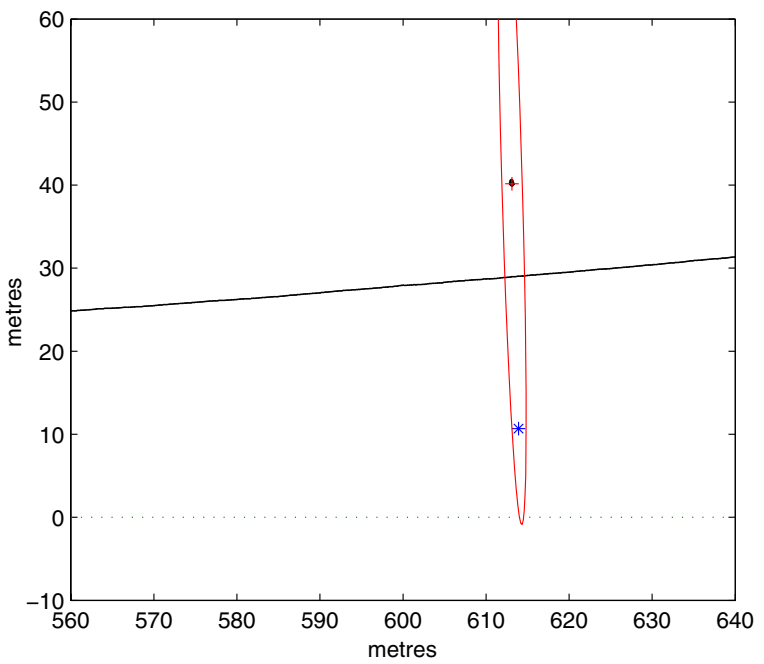

(b) Ideal Jacobians.

Fig. 4. Jagged vehicle path and linear feature locus are artifacts of the inconsistency induced by variations in Jacobian linearisation given large heading uncertainty. These figures depict a section of two runs, which are identical apart from the Jacobian computation. The true vehicle trajectory is along the $\mathrm{x}$-axis (i.e., $y=0$ ), the true landmark location is indicated by a $*$.

A measure of filter consistency is found by examination of the average NEES over N Monte Carlo runs of the filter. ${ }^{6}$ Under the hypothesis that the filter is consistent and approximately linear-Gaussian, $\epsilon_{k}$ is $\chi^{2}$ (chi-square) distributed with $\operatorname{dim}\left(\mathbf{x}_{k}\right)$ degrees of freedom. Then the average value of $\epsilon_{k}$ tends towards the dimension of the state as $N$ approaches infinity.

$$
E\left[\epsilon_{k}\right]=\operatorname{dim}\left(\mathbf{x}_{k}\right)
$$

${ }^{6}$ Of SLAM papers that discuss filter errors, the great majority, including [10] and [4], present single run results. However, single run NEES is an inadequate measure of filter consistency, as the error sequence is correlated and does not follow a $\chi^{2}$ distribution. In fact, it is simple to generate a single Monte Carlo run of a consistent linear filter where the NEES sequence lies outside a $95 \%$ bound for most of the run. Conversely, it is also possible to obtain a single run of an inconsistent filter that appears consistent. To quote Bar-Shalom [2, page 394]: “. . a thorough examination of a nonlinear filter is needed via [multiple] Monte Carlo runs to find out if it is consistent". 
The validity of this hypothesis can be subjected to a $\chi^{2}$ acceptance test.

Consistency of the EKF is evaluated by performing multiple Monte Carlo runs and computing the average NEES. Given $N$ runs, the average NEES is computed as

$$
\bar{\epsilon}_{k}=\frac{1}{N} \sum_{i=1}^{N} \epsilon_{i_{k}}
$$

Given the hypothesis of a consistent linear-Gaussian filter, $N \bar{\epsilon}_{k}$ has a $\chi^{2}$ density with $N \operatorname{dim}\left(\mathbf{x}_{k}\right)$ degrees of freedom [2, pages 234-235]. Thus, for the 3-dimensional vehicle pose, with $N=$ 50 , the $95 \%$ probability concentration region for $\bar{\epsilon}_{k}$ is bounded by the interval $[2.36,3.72]$. If $\bar{\epsilon}_{k}$ rises significantly higher than the upper bound, the filter is optimistic, if it tends below the lower bound, the filter is conservative.

We tested the average NEES consistency of four variations of the EKF-SLAM algorithm: a basic EKF, an iterated EKF (IEKF) [2], an unscented Kalman filter (UKF) [9] and an EKF with ideal Jacobians. ${ }^{7}$ Each filter was run for two loops of the trajectory shown in Fig. 2, and each Monte Carlo simulation was repeated 50 times. The results are shown in Fig. 5. Clearly the EKF, IEKF and UKF all become optimistic after about 100 seconds. ${ }^{8}$ The EKF also exhibits a large spike at about 220 seconds when it closes the loop. The UKF shares this characteristic, but the IEKF does not. It appears that the IEKF can improve loop closing results since the initial state error is typically very large and linearisation benefits from iteration. However, when not closing the loop, the IEKF offers no advantage as the SLAM state is not locally observable, and iteration may move the estimated mean away from the true state, actually enhancing divergence. The UKF is not a panacea but appears to degrade more slowly than the EKF. Its advantages are probably more pronounced for very non-linear models.

The failure of EKF-SLAM in Fig. 5(a-c) is unavoidable; adding stabilising noise to the process and observation models does not improve the result. However, this problem does not arise if the true heading uncertainty remains small. Running the basic EKF with regular observations of heading, so that the true heading uncertainty is always small, produces consistent results. This result is insensitive to the system noises. For example, we performed experiments with process noise $\left(\sigma_{V}=1.0 \mathrm{~m} / \mathrm{s}, \sigma_{\gamma}=5^{\circ}\right)$ and observation noise $\left(\sigma_{r}=1.0 \mathrm{~m}, \sigma_{\theta}=10^{\circ}\right)$, and obtained estimates within the upper/lower NEES bounds, similar to Fig. 5(d). Thus, with small heading uncertainty, EKF-SLAM does not suffer abrupt catastrophic inconsistency.

When heading uncertainty remains small, EKF-SLAM exhibits inconsistency of a qualitatively different kind. We performed a set of simulations in an environment of 60 by 70 metres containing 15 landmarks, which was sufficiently small

\footnotetext{
${ }^{7}$ MATLAB source code for these simulations can be obtained from: www.acfr.usyd.edu.au/homepages/academic/tbailey/software/index.html.

${ }^{8}$ Notice that the rapid rise in NEES coincides roughly with the start of significant information gain in Fig. 3.
}

that the vehicle heading standard deviation never exceeded one degree. For each run, the vehicle traversed 100 loops of the environment, and this was repeated 50 times. The 50-run average NEES of the vehicle pose states is shown in Fig. 6(a) and the average NEES of the joint state of two landmarks is shown in Fig. 6(b). ${ }^{9}$ Both figures show a slow steady increase in NEES over time, so that the filter eventually becomes optimistic (at around 4000 seconds, say).

A UKF implementation of this experiment gave minor improvement, as shown in Fig. 6(c). We were able to temporarily suppress optimistic estimates by artificially raising the covariances of the process and observation noise models. In Fig. 6(d), the small-scale EKF experiment was repeated with the noise variance models inflated by a factor of two. The average NEES still increases with time, but we project that it would not cross the upper bound until about 25000 seconds.

From the results in Figs. 5 and 6, the effect of heading variance is clear. The large-variance behaviour is abrupt inconsistency, as seen by the change in slope at around 100 seconds in Fig. 5(a). This failure is not prevented by stabilising noise. The small-variance behaviour, on the other hand, is a slow steady rise in NEES. Failure by way of optimistic estimates is time dependent, and can be delayed by adding stabilising noise. These results agree with our expectation from the stationary vehicle experiments in Section III-A, where rate of inconsistency is a non-linear function of heading variance.

\section{Discussion}

Previous investigations of EKF-SLAM consistency claim that the algorithm's failure is time dependent [10], [4]. Our results agree with this, but we claim that a more significant source of inconsistency is heading variance which, if large, can precipitate failure in just a few updates. In particular, we note that the inconsistencies described in [10] and [4] are almost certainly due to growing heading uncertainty, not time. Time dependent failure occurs only if heading variance remains small, and then conservative estimation may be prolonged by adding stabilising noise. ${ }^{10}$ Our long-run small-scale experiments suggest that one should eventually cease data fusion and freeze the map to prevent optimism.

Many of the arguments against the EKF-SLAM algorithm do not address the real problem. For example, two common arguments claim it is inadequate because (i) it poorly linearises the process and observation models, and (ii) it cannot consistently "close the loop".

With regard to (i), our UKF and IEKF experiments show that improved local linearisation does not greatly improve consistency. Linearisation must be congruous on a more global scale to be of significant benefit. Likely candidates are EKF-SLAM variants that minimise heading uncertainty and

\footnotetext{
${ }^{9}$ We expect that Fig. 6(b) would be characteristic of any two landmarks, but the landmarks in question were located on opposite sides of the map, at $(41,-10)$ and $(-35,16)$.

${ }^{10}$ While, arguably, an overconservative estimate is as bad as an optimistic one in terms of convergence, a conservative estimate is crucial for safe decision making, such as data association.
} 


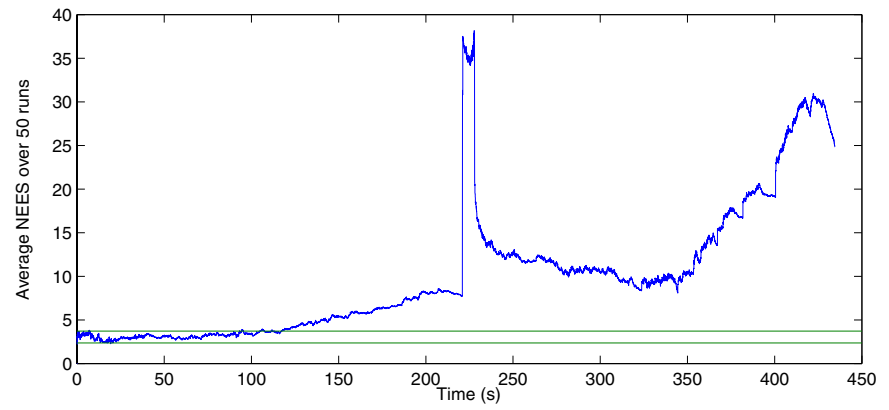

(a) EKF

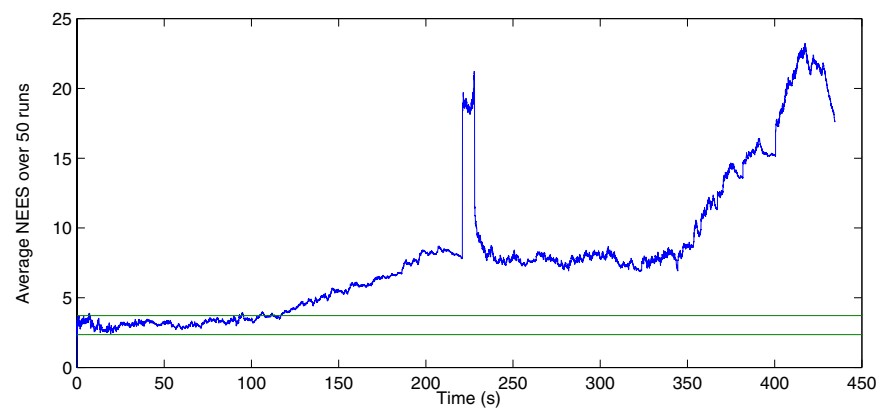

(c) UKF

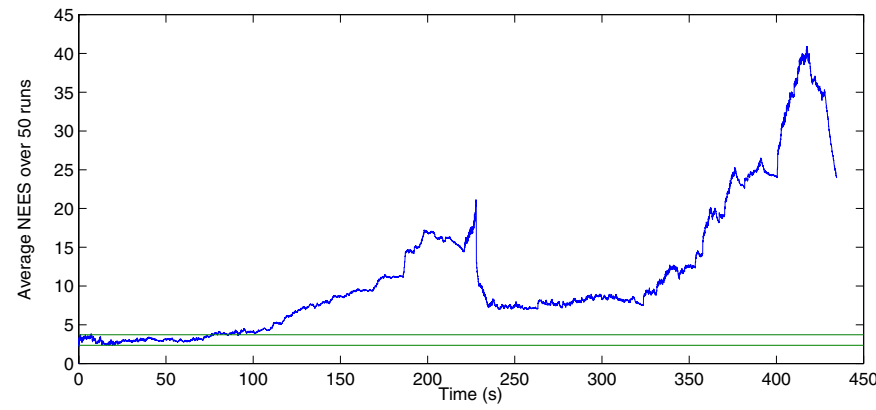

(b) IEKF

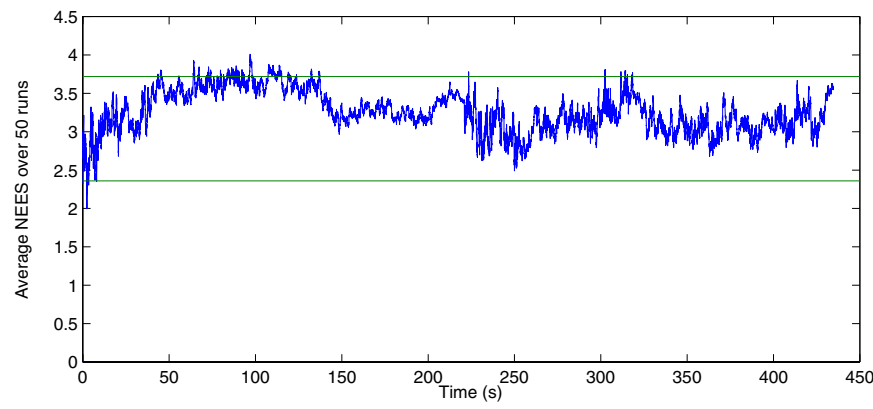

(d) EKF with ideal Jacobians

Fig. 5. Average NEES of the vehicle pose states $\left[x_{v_{k}}, y_{v_{k}}, \phi_{v_{k}}\right]^{T}$ over 50 Monte Carlo runs. The horizontal lines mark the $95 \%$ probability concentration region for a 3-dimensional state vector. Only the EKF with ideal Jacobians remains consistent.

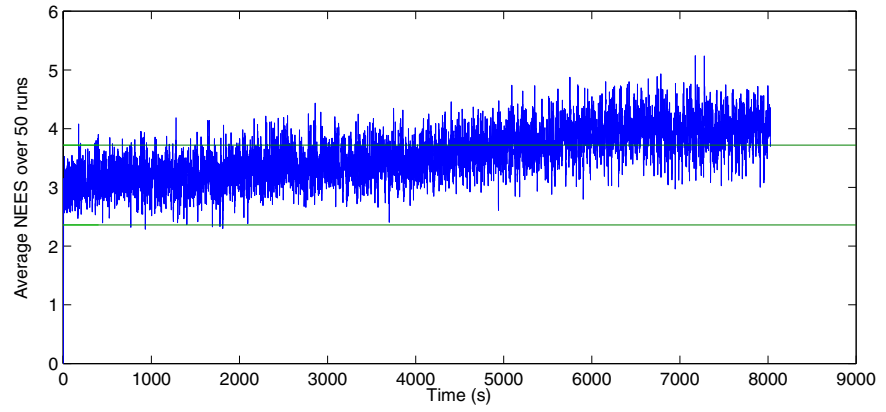

(a) EKF, vehicle pose $\left[x_{v_{k}}, y_{v_{k}}, \phi_{v_{k}}\right]^{T}$

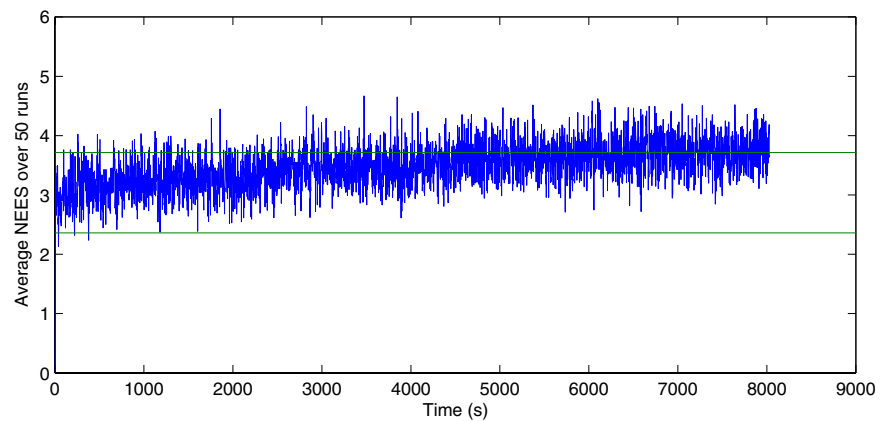

(c) UKF, vehicle pose

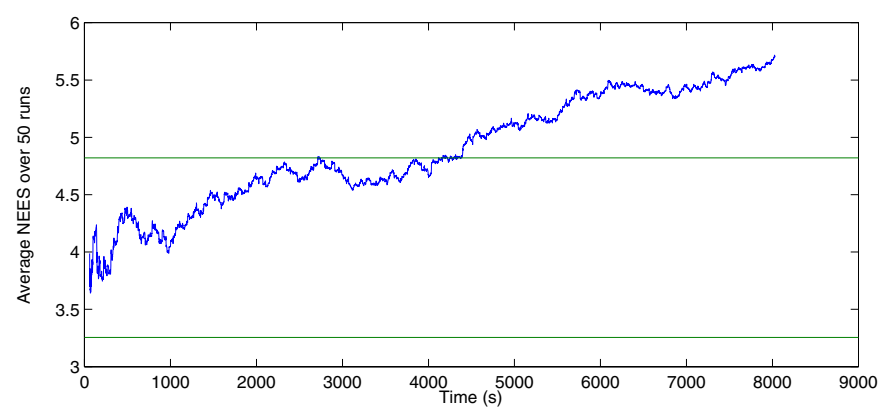

(b) EKF, two landmarks $\left[x_{i}, y_{i}, x_{j}, y_{j}\right]^{T}$

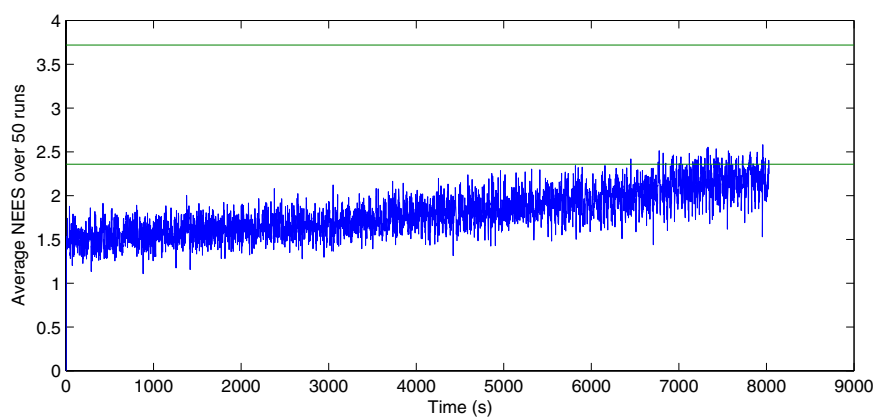

(d) EKF with stabilising noise, vehicle pose

Fig. 6. Long-term behaviour of EKF-SLAM for small-scale mapping. Average NEES of (a) the vehicle pose states and (b) two landmark states over 50 Monte Carlo runs. Two attempts to improve consistency are shown in (c) and (d). 
minimise variations in Jacobian linearisation. The constrained local submap filter (CLSF) [16] is one such method as it performs most updates in a local frame with small heading uncertainty. Batch update methods, such as fixed-interval smoothing between timesteps $k$ and $k+N$, are also likely to help.

With regard to (ii), the failure to properly close large loops experienced in practice is probably from the SLAM estimate already being inconsistent due to heading uncertainty. If the state is consistent prior to closing the loop, then a consistent update depends entirely on linearising the measurement model, which suggests loop-closing is well suited to an IEKF update.

For very large-scale maps, submap methods, such as network coupled feature maps (NCFM) [1] and ATLAS [3], are currently the only solutions that avoid data-fusion with large heading variance. These submap methods confine all updates to small-scale maps where the heading variance is small. The network of submaps permits consistent global estimation as a sum of local-frame connections, avoiding ever making a global-level Kalman update. Thus, at a large-scale, spurious information gain is no worse than for a small-scale map.

\section{CONCLUSION}

In [10, Section 3], Julier and Ulhmann proves that the non-linear models in EKF-SLAM introduce spurious updates of the landmark estimates, for any finite noise values, due to variations in linearisation. The algorithm is, therefore, theoretically inconsistent under all circumstances.

In this paper, we show that inconsistency is not always dire, and its seriousness is directly related to heading uncertainty. If the true heading variance is large, failure is sudden and irredeemable but, if it remains small throughout, inconsistency grows slowly and is manageable. This is expected behaviour as our stationary vehicle experiments show a highly non-linear relationship between heading variance and the quantity of spurious information gained from a measurement update.

A problem for real EKF-SLAM implementations is that failure due to "large" heading variance is subtle and difficult to detect without ground-truth. In our moving vehicle experiments, we found that significant error in estimated heading uncertainty began once the true heading uncertainty exceeded about 1.7 degrees (std. dev.). The filter, however, gave no indication of anything amiss; the estimated variance simply plateaus while the "true" variance continues to grow. An observable symptom that may occur, once the estimate has become very inconsistent, is a "jagged" vehicle path, where the vehicle pose update is significantly greater than would be expected from the actual measurement error.

Inconsistency can be prevented if the Jacobians for the process and observation models are always linearised about the true state. For standard EKF linearisation, inconsistency accumulates slowly while the vehicle heading uncertainty remains small, and the filter is robust to process/observation noises and non-linearities, and is assisted by stabilising noise. SLAM is stable for long periods under these conditions.
The IEKF and UKF cannot prevent inconsistency. They offer no fundamental improvement over plain EKF-SLAM. Batch update methods are likely to extend the region over which EKF-SLAM can construct a consistent global map as they reduce local heading uncertainty and Jacobian variation. For large-scale maps, submaps confine EKF updates to the small-scale, and prevent any data fusion with large heading uncertainty. Submaps are currently the only way to implement large-scale EKF-SLAM without excessive information gain.

\section{ACKNOWLEDGEMENTS}

Thanks to Simon Julier for an insightful discussion on the long-term behaviour of small-map SLAM. This work is supported by the ARC Centre of Excellence programme, funded by the Australian Research Council (ARC) and the New South Wales State Government.

\section{REFERENCES}

[1] T. Bailey. Mobile Robot Localisation and Mapping in Extensive Outdoor Environments. PhD thesis, University of Sydney, Australian Centre for Field Robotics, 2002.

[2] Y. Bar-Shalom, X.R. Li, and T. Kirubarajan. Estimation with Applications to Tracking and Navigation. John Wiley and Sons, 2001.

[3] M. Bosse, P. Newman, J. Leonard, M. Soika, W. Feiten, and S. Teller An Atlas framework for scalable mapping. In IEEE International Conference on Robotics and Automation, pages 1899-1906, 2003.

[4] J.A. Castellanos, J. Neira, and J.D. Tardós. Limits to the consistency of EKF-based SLAM. In IFAC Symposium on Intelligent Autonomous Vehicles, 2004.

[5] M.W.M.G. Dissanayake, P. Newman, S. Clark, H.F. Durrant-Whyte, and M. Csorba. A solution to the simultaneous localization and map building (SLAM) problem. IEEE Transactions on Robotics and Automation, 17(3):229-241, 2001.

[6] J. Guivant and E. Nebot. Improving computational and memory requirements of simultaneous localization and map building algorithms. In IEEE International Conference on Robotics and Automation, pages 2731-2736, 2002.

[7] J.S. Gutmann and K. Konolige. Incremental mapping of large cyclic environments. In IEEE International Symposium on Computational Intelligence in Robotics and Automation, pages 318-325, 1999.

[8] S. Huang and G. Dissanayake. Convergence analysis for extended Kalman filter based SLAM. In IEEE International Conference on Robotics and Automation, 2006.

[9] S. Julier, J. Uhlmann, and H.F. Durrant-Whyte. A new method for the nonlinear transformation of means and covariances in filters and estimators. IEEE Transactions on Automatic Control, 45(3):477-482, 2000.

[10] S.J. Julier and J.K. Uhlmann. A counter example to the theory of simultaneous localization and map building. In IEEE International Conference on Robotics and Automation, pages 4238-4243, 2001.

[11] S.J. Julier and J.K. Uhlmann. Using multiple SLAM algorithms. In IEEE/RSJ International Conference on Intelligent Robots and Systems, pages 200-205, 2003.

[12] J. Leonard and P. Newman. Consistent, convergent, and constant-time SLAM. In International Joint Conference on Artificial Intelligence, 2003.

[13] M. Montemerlo, S. Thrun, D. Koller, and B. Wegbreit. FastSLAM 2.0: An improved particle filtering algorithm for simultaneous localization and mapping that provably converges. In International Joint Conference on Artificial Intelligence, pages 1151-1156, 2003.

[14] J. Neira, J.D. Tardós, and J.A. Castellanos. Linear time vehicle relocation in SLAM. In IEEE International Conference on Robotics and Automation, 2003.

[15] R. Smith, M. Self, and P. Cheeseman. A stochastic map for uncertain spatial relationships. In International Symposium of Robotics Research, pages 467-474, 1987.

[16] S.B. Williams. Efficient Solutions to Autonomous Mapping and Navigation Problems. PhD thesis, University of Sydney, Australian Centre for Field Robotics, 2001. 